\title{
The effect of a moving dot transversal on the Poggendorff illusion
}

\author{
MARK B. FINEMAN and MARK P. MELINGONIS \\ Southern Connecticut State College, New Haven, Connecticut 06515
}

\begin{abstract}
In attempting to derive the minimal component of the Poggendorff figure which would still produce an illusion, responses to three types of transversal were measured. One was the customary solid line type; a second type presented the two segments sequentially, alternating between them; and the third consisted of a moving dot which traveled the transversal path. Each transversal was shown with and without verticals, for a total of six conditions. Ten subjects in each condition adjusted the luminous transversal until the segments appeared to be collinear. Figures with verticals present showed a greater magnitude of illusion than those without, and discrepancies for moving dot transversals were greater than those for comparable solid line figures. Since alternating transversals were not significantly larger than solid line figures, it was concluded that the magnitude of the moving dot effect could not be attributed to temporal sequence. An eyemovement hypothesis was suggested instead.
\end{abstract}

The Poggendorff illusion (Burmester, 1896) consists of two parallel, vertical lines intersected by two oblique lines lying along the same straight line. The oblique lines are sometimes referred to as the diagonal or transversal elements of the figure; the illusion is derived from the observation that most people report the two portions of the transversal to be displaced from true geometric alignment.

A time-honored way of studying the illusion, in fact virtually all geometric illusions, has been to analyze the relative contributions of various components of the figure. Wundt (1893) was among the first to do this, claiming that it was the orientation of the transversal alone that gave rise to the illusion of discontinuity. More recently, Krantz and Weintraub (1973) and Weintraub and Krantz (1971) measured the relative contributions of the vertical and transversal elements; Day (1973), Farné (1970), and Goldstein and Weintraub (1972), all measured the magnitude of the illusion with no verticals present. These studies may be summarized by saying that a small, sometimes significant, illusion has been obtained by several investigators using a figure with no verticals. One might also describe such displays as degradations of the original Poggendorff figure.

Coren (1970) measured a different sort of degraded figure, one in which dots at the endpoints of the lines were substituted for the lines themselves. It was reported that this configuration produced less of an illusion than the complete figure, but still an illusion greater than zero. Without exception,

Requests for reprints may be sent to: Mark B. Fineman, Southern Connecticut State College, Department of Psychology, 501 Crescent Street, New Haven, Connecticut 06515. previous researchers report a reduction in the magnitude of the illusion with increasing degradations of the Poggendorff figure.

Our investigations grew out of an interest in finding the minimal component of the original figure which would still produce an illusion. Coren's figure, while not composed of continuous contours, nonetheless retained the vertical elements; Wundt's figure, and others like it, did not use verticals but still contained continuous line transversals. While the obvious resolution of this difference might have been to measure judgments of diagonally oriented dots, we elected instead to see if a single dot moving along the path normally described by the transversal would induce the illusion. We felt that this stimulus condition might be even more impoverished than those of previous researchers.

Because previous studies had shown that extreme degradations of the Poggendorff figure produced little or no illusion, we decided to also include a condition in which verticals would be present, as in the traditional figure, but with a moving dot transversal. It was felt that should an effect be obtained the result would still be of interest, particularly in light of how such a finding might bear on several theories of the geometric illusions.

Both the dot transversal condition and the dot transversal with verticals condition were to be compared with solid line figures, one of which was a transversal-only display, the other of which included verticals (the Poggendorff figure). Since the judgments of the first few subjects clearly showed an illusion for both moving dot figures, it was decided to include two additional conditions in which solid line transversal segments would appear alternately. The two versions were one in which the two diagonal 
segments alone appeared alternately, and one with verticals present as well. The reason for including the alternating transversal figures was to test the temporal property of the transversal in the absence of movement, a point which will be considered at greater length below.

Thus the experiment took the form of a completely randomized factorial design (Linton \& Gallo, 1975). The first independent variable, the type of transversal, was presented at three levels (solid, moving dot, and alternating), and the second independent variable, the vertical component, was presented at two levels (verticals present and verticals absent), for a total of six conditions.

\section{METHOD}

\section{Subjects}

Ten subjects were assigned to each condition, 60 subjects in all. They were undergraduate students ( 19 males and 41 females) attending Southern Connecticut State College, and all were unpaid volunteers.

\section{Apparatus}

Because only half of the conditions included vertical lines and several involved motion, it was decided to present the verticals in a device separate from that producing the transversals. Luminous transversals were generated in a box-like device $(39.5 \times 33 \times 15 \mathrm{~cm})$ directly in front of the subject. In every condition, the brightness of the transversal was made to appear equal to that of the verticals.

Solid transversal. The transversal segments were cut from the thin opaque cardboard sheet that covered the front of the stimulus box. The stimulus dimensions are summarized in Figure 1. A 7-W incandescent lamp within the box illuminated the transversal slits on the front of the box. Two layers of white bond paper were glued to the rear of the slits to produce uniformly illuminated lines. The left half of the transversal (Segment A, Figure 1) was stationary, but the right half (Segment B, Figure 1) was on a movable cardboard slide, held down within a vertical track, which could be moved up or down by means of a continuous nylon line attached to the top and bottom of the slide. A vertical millimeter scale was affixed to the face of the display between the transversal segments, and was marked in such a way that when the left terminus of Segment B was at the zero point it was physically aligned with Segment A; deviations from collinearity were positive or negative in relation to the scale's zero point. Except for the manner of illumination, this device was used for all conditions.

Moving dot transversal. A moving spot of light was made to travel the length of the diagonal slits by interposing a rotating disk between the 7-W light source and the apertures on the front of the box. The disk measured $22.3 \mathrm{~cm}$ in diameter and had a $1 \times$ $200 \mathrm{~mm}$ slit cut from it, centered on its diameter. It was driven by a dc timing motor at $10 \mathrm{rpm}$, so that the moving spot of light appeared to travel the length of the complete transversal 20 times/min. While this arrangement did not produce a dot which moved at a completely uniform velocity, variations in the acceleration of the dot were symmetrical about the center of the transversal slit and were of an extremely small magnitude.

Alternating transversal. The two portions of the transversal were now illuminated by two 7-W light sources encased in separate compartments within the box; each lamp illuminated a single segment exclusively. The lamps were turned on and off by microswitches regulated by a motor-driven cam timer in the same sequence and for the same duration as in the moving dot conditions. The on-off sequence was: Segment $A$ on for $0.375 \mathrm{sec}$,

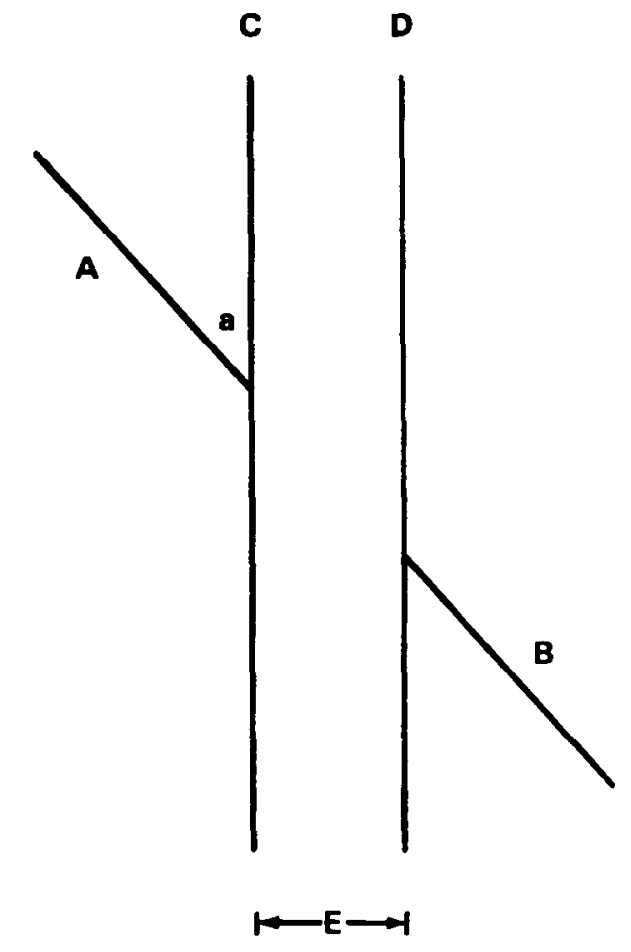

Figure 1. The composite Poggendorff figure used in this experiment: transversal segment $A=40 \mathrm{~mm}$, transversal segment $B=40 \mathrm{~mm}$, vertical $C=100 \mathrm{~mm}$, vertical $D=100 \mathrm{~mm}$, $E=19 \mathrm{~mm}$, and $\mathrm{a}=40 \mathrm{deg}$.

both segments off for $0.75 \mathrm{sec}$, Segment $\mathrm{B}$ on for $0.375 \mathrm{sec}$, both segments off for $1.50 \mathrm{sec}$. In this way, the slits were illuminated exactly as they had been for moving dot transversals, but with a continuous stimulus, as with the solid transversal.

Vertical lines. A second stimulus box, essentially identical to the one described for generating the transversals, was located to the subject's right. This, too, was illuminated by a 7-W incandescent lamp and had an opaque cardboard mask affixed to its front. Two vertical lines (Segments C and D, Figure 1) were cut from the mask, and a single thickness of white bond paper was glued to the back of the mask so that the lines would be uniformly illuminated. Composite displays (for the three conditions with both verticals and transversals) were viewed by means of a beam splitter which was located $16.5 \mathrm{~cm}$ in front of the subject's right eye and $35 \mathrm{~cm}$ from each display. Thus, the transversal display was observed through the glass, while the vertical display was seen as a reflection. The beam splitter itself was a 1 -mm-thick glass plate $(82 \times 102 \mathrm{~mm})$ held stationary in an optical bench.

\section{Procedure}

The subjects had been told that the experiment was a study dealing with the way people judge the position of lines. Before a subject entered the laboratory, the experimenter described the general appearance of the display and then told the subject that his task would be to adjust the two diagonal lines until they appeared to lie along the same straight line. If the subject normally wore corrective lenses, he was told to continue wearing them for the duration of the experiment. The subject's left eye was occluded with an eyepatch so that all observations would be made with the right eye. This part of the procedure was necessary because pilot studies had shown that even when the beam splitter was equidistant from the vertical and transversal stimuli, there was still a tendency to see the two parts at slightly 
different distances. Monocular viewing eliminated disparity cues, and, in fact, the subjects never reported that the composite display appeared disjointed.

The subject was then asked to close his eyes, was led into the darkened room, and seated at the apparatus. His head was held stationary in a chin and headrest. The continuous nylon line attached to the top and bottom of the Segment B slide was placed in the subject's hands and the method of adjustment explained. Each subject viewed only one of the six conditions and was required to make four adjustments of the right diagonal segment. For each subject, there were two ascending and two descending trials, in different orders, the mean of which was used as the subject's score. No time limits were imposed.

\section{RESULTS}

The mean error score for the 10 subjects in each condition is shown in Table 1 . In every case, the error is positive and, except one, significantly greater than zero. It can also be seen that within diagonal type the presence of vertical lines increased the magnitude of the illusion. An analysis of variance indicated significance for type of transversal $(F=12.30, \mathrm{df}=2,54, \mathrm{p}<.01)$ and presence or absence of verticals $(F=30.28$, $\mathrm{df}=1,54, \mathrm{p}<.01$ ), but not for the interaction of the two variables.

Comparisons of individual means showed that the transversal effects were attributable to a significant difference between the solid transversal and dot transversal conditions $(p<.01)$ and the dot transversal and alternating transversal conditions $(p<.01)$; all other comparisons between transversal conditions were not significant.

\section{DISCUSSION}

The moving dot conditions not only produced the illusion, but an illusion which exceeded comparable solid line figures. This finding is, of course, an exception to the general rule that the magnitude of the illusion decreases with impoverishment of the Poggendorff figure. That the illusion even exists for moving dot transversals poses problems for any theory which proposes that the illusion occurs because of the way lines interact. In the case of the moving dots, there are no lines as such. Coren's (1970) work was also meant to show that lines are not necessary for the Poggendorff illusion to occur, but another interpretation might simply be that "line" may be defined in several ways, e.g., a series of dots, subjective contour (Gregory, 1972), or, in this case, a single moving dot. Thus, while the results reported here do not necessarily rule out a lateral inhibition model, or any other that describes line interaction, they certainly call for more sophisticated statements of those theories.

Naturally, we wondered why the moving dot was more effective at producing the illusion than solid line figures. When this effect was first noted, we hypothesized that the difference might be attribut-
Table 1

Mean Error in Millimeters and Standard Deviation for Each Display

\begin{tabular}{cccc}
\hline & \multicolumn{3}{c}{ Type of Transversal } \\
\cline { 2 - 4 } Vertical Component & Solid & Alternating & Moving Dot \\
\hline Verticals & 1.20 & 1.28 & 6.35 \\
Absent & $(.58)$ & $(4.29)$ & $(2.20)$ \\
Verticals & 5.37 & 6.87 & 8.48 \\
Present & $(1.79)$ & $(3.23)$ & $(2.21)$ \\
\hline
\end{tabular}

able to the temporal property of the moving dot. Since Segment A was not illuminated simultaneously with Segment $B$ in the moving dot conditions, perhaps a memory effect was at work: that the two segments were not presented simultaneously might have led to increased errors of judgment. The alternating segments were like the solid line transversal in composition but more closely resembled the dot transversal in temporal sequence.

Considering just those conditions with verticals absent (see Table 1), it can be seen that the alternating transversal condition was nearly identical to the solid line condition but that the moving dot condition was significantly larger than the alternating condition $(p<.01)$. This says, in effect, that the increased magnitude of the illusion with the moving dot transversal may not be attributable to the temporal property of the moving dot. In the absence of verticals, both solid line figures produced extremely small effects. Taken singly, the solid transversal effect was just barely significant and the alternating transversal was not significant (both at .05). These results are remarkably similar to those obtained by Goldstein and Weintraub (1972); such small effects might be attributable to measurement errors. Should the level of the illusion for these two solid transversal figures prove to be artifactual, then the moving dot transversal illusion may well be a new effect.

Although the three conditions with verticals present followed the same trend as those without verticals, no significant differences were obtained. The finding that the addition of verticals significantly increases the magnitude of the illusion is totally consistent with other research, and it may have been the case that the addition of the verticals overpowered the smaller transversal alone effect or it may simply be that solid transversals without verticals produce no reliable illusion. Since it is conceptually easier to compare figures having no verticals, we must conclude that the temporal property of the moving dot transversal does not seem to account for the increased magnitude of the illusion over a solid line figure.

Only one other hypothesis readily suggests itself, a hypothesis which has yet to be tested. Perhaps the moving dot increased the rate of trajectory eye movements and the eye movements in some way account 
for the difference between solid line and moving dot judgments. This is not to say that eye movements cause the illusion-ample evidence contradicts that notion-only that they play a role in the magnitude of the illusion. For example, Novak (1966) reported that when subjects were required to fixate a marker at the center of the Poggendorff figure, the magnitude of the illusion was less than with free inspection of the same figure. Although this suggests that eye movements have something to do with the magnitude of the illusion, future research should clarify whether a specific type of eye movement contributes to the effect (which we suggest), or if it is simply due to the total number of eye movements. The latter notion would seem to be contradicted by the wellestablished finding that prolonged inspection decreases the magnitude of the illusion (Judd, 1902).

\section{REFERENCES}

BURMESTER, E. Beitrage zur experimentellen Bestimmung geometrisch-optischer Täuschungen. Zeitschrift für Psychologie, $1896,12,355-394$.

COREN, S. Lateral inhibition and geometric illusions. Quarterly Journal of Experimental Psychology, 1970, 22, 274-278.
DAY, R. H. The oblique line illusion: The Poggendorff effect without parallels. Quarterly Journal of Experimental Psychology, $1973,25,535-541$.

FarNÉ, M. On the Poggendorff illusion: A note to Cumming's criticism of Chung Chiang's theory. Perception \& Psychophysics, $1970,8,112$.

Goldstein, M. B., \& Weintraub, D. J. The parallel-less Poggendorff: Virtual contours put the illusion down but not out. Perception \& Psychophysics, 1972, 11, 353-355.

Gregory, R. L. Cognitive contours. Nature, 1972, 238, 51-52.

Judd, C. H. Practice and its effects on the geometrical illusions. Psychological Review, 1902, 9, 27-39.

Krantz, D. H., \& Weintraub, D. J. Factors affecting perceived orientation of the Poggendorff transversal. Perception \& Psychophysics, 1973, 14, 511-517.

Linton, M., \& Gallo, P. S. The practical statistician: Simplified handbook of statistics. Monterey, Calif: Brooks/Cole, 1975.

Novak, S. Effects of free inspection and fixation on the Poggendorff illusion. Perceptual and Motor Skills, 1966, 23, 663-670.

Weintraub, D. J., \& Krantz, D. H. The Poggendorff illusion: Amputations, rotations, and other perturbations. Perception \& Psychophysics, 1971, 10, 257-264.

WundT, W. Grundzüge der physiologischen Psychologie. Leipzig: Englemann, 1893.

(Received for publication August 23, 1976; revision received November $22,1976$. 